Pérez Asperilla, Estíbaliz.

Investigadora, Universidad Complutense de Madrid, Departamento de Comunicación Audiovisual y Publicidad II, Grupo de Investigación Arte, Arquitectura y Comunicación en la Ciudad Contemporánea.

\title{
Transformando Bankside.
}

\section{Transforming Bankside.}

\author{
TIPO DE TRABAJO: \\ Comunicación virtual.
}

PALABRAS CLAVE:

Bankside, transformación, participación ciudadana, Distrito Cultural.

KEY WORDS:

Bankside, transformation, public participation, Cultural District.

RESUMEN.

Bankside supone un claro ejemplo de revitalización urbana puesto que, a pesar de haber sido considerado como una de las zonas más contaminadas de Londres, ha logrado transformarse en un Distrito Cultural mundialmente conocido gracias a la apuesta e inversión en diferentes creaciones artísticas. No sólo se han rehabilitado edificios vacíos y en desuso dando lugar a centros artísticos de suma importancia como es el caso de la conversión de su antigua central eléctrica en lo que ahora se conoce como la Tate Modern - una de las galerías de arte contemporáneo más importantes de Europa -, sino que el arte sale a la calle ilustrando muros, fachadas y dando vida a zonas que aún se encuentran a la espera de su próxima regeneración. La ciudad envuelve al ciudadano/a por completo ofreciendo un Distrito Cultural que se reinventa constantemente dando la posibilidad de que el público no sea un mero espectador, sino de que se sumerja en la historia y cultura del Distrito convirtiéndose a su vez en creador y artista e invitándole a formar parte de las actividades que se desarrollan en diferentes festivales como Merge o el London Design Festival. Muchas de las instalaciones y obras de arte son temporales y/o perecederas. No obstante, algunas de las obras que florecen a lo largo de sus calles y plazas se han ganado su permanencia, tal como sucede con las realizadas alrededor del santuario de the Crossbones Graveyard.

Esta comunicación se enmarca dentro de la investigación Los cinco sentidos del Distrito Cultural en Londres, la cual forma parte del Proyecto de Excelencia 2016-2018 Distritos Culturales: imágenes e imaginarios en los procesos de revitalización de espacios urbanos (Ref. HAR2015-66288-C4-2-P, Plan Nacional I+D).

\section{ABSTRACT}

Bankside is a clear example of urban revitalization since, despite being considered one of the most polluted areas of London, it has managed to transform itself into a world-famous Cultural District thanks to the bet and investment in different artistic creations. Not only empty and disused buildings have been rehabilitated, giving rise to important artistic centers such as the conversion of its former power station into what is now known as the Tate Modern - one of the most important contemporary art galleries of Europe -, but art goes out illustrating walls, facades and giving life to areas that are still waiting for their next regeneration. The city involves citizens by offering a Cultural District that reinvents itself constantly giving them the possibility of being not mere spectators, but that they immerse themselves into the history and culture of the District becoming themselves creators and artists and inviting them to form part of the activities that take place in different festivals such as Merge or London Design Festival. Many of the installations and works are temporary and/or perishable. Nevertheless, some of the works that flourish along its streets and squares have gained their permanence like the sanctuary of the Crossbones Graveyard. 
This paper is part of the research Five senses of London Cultural District, which forms part of the Excellence Project 20162018 Cultural Districts: images and imaginaries in the revitalization process of urban locations (Ref. HAR2015-66288-C4-2-P, I+D National Plan).

\section{CONTENIDO.}

\section{INTRODUCCIÓN.}

Considerado originariamente como el barrio del pecado al caracterizarse no sólo por los numerosos burdeles, teatros y tabernas que se localizaron en la zona, sino por la gran cantidad de malhechores que empezaron a inundar el lugar, Bankside ha experimentado una regeneración constante tras la gran desindustrialización que desoló la ciudad de Londres. Regeneración que sigue en marcha gracias a las acciones que diferentes festivales y organizaciones llevan a cabo teniendo como uno de sus pilares clave la conservación de su historia, patrimonio y cultura. La fusión entre el pasado y el presente permite que la comunidad local conozca los orígenes del Distrito mediante técnicas innovadoras e instalaciones participativas considerando a su vez al público como sujeto indispensable.

"The diversity of people that live in it is part of the essence of Bankside. Benefiting of historical waves o change and migration, the presence of different skin colours, tongues, occupations, lifestyles and bank balances may be the reason why so many residents feel

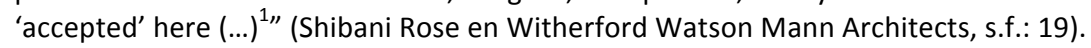

A continuación se han seleccionado algunas de las acciones que han tenido lugar en los últimos años comprobando así cómo las instalaciones se fusionan con la historia del Distrito y cómo la cultura y el patrimonio de este último enriquece las obras tanto de los artistas emergentes como de los ya establecidos optando por la conservación de la esencia de Bankside como eje principal.

\section{BEFORE I DIE Y SIDEWALK PSYCHIATRY.}

Ubicando varias pizarras en las localizaciones de Flat Iron Square, Union Street y Borough High Street, Candy Chang volvió a recrear el proyecto Before I die ${ }^{2}$ que originariamente tuvo lugar en New Orleands tras haber sufrido la pérdida de un ser querido. Al reflexionar sobre la muerte y lo que realmente importaba en su vida, puso en marcha este proyecto invitando a la gente a participar de una forma activa al completar la frase que escribió en repetidas ocasiones sobre la pizarra - "Before I die I want to..." ${ }^{3}$. No sólo tuvo una gran acogida en New Orleands, sino también en el Distrito que nos ocupa durante Merge 2013. Al utilizar tizas, el contenido de las pizarras se renovaba constantemente ofreciendo así una instalación cambiante, creativa y participativa donde tanto los habitantes de Bankside como la gente de paso eran indispensables para su creación y permanencia. "(...) this neglected space became a constructive one (...). It's about you're not alone; it's about understanding our neighbors in new and enlightening ways; it's about making space for reflection and contemplation, and remembering what really matters most to us as we grow and change ${ }^{4 \prime}$ (Chang, 2012).

Tras el gran éxito de Before I Die, Candy Chang volvió a participar en Merge del año 2014 con Sidewalk Psychiatry. Escribiendo diez preguntas dirigidas a la auto reflexión del individuo hizo posible que el Distrito se comunicara con los/las viandantes que paseaban por sus calles planteándoles cuestiones como ¿qué estás esperando? ; ¿piensas que fue bien?; ¿entonces por qué lo haces?; ¿qué quieres?; ¿saben cómo te sientes? De esta forma, ya fuera camino del trabajo o de vuelta a casa el público se topaba con una llamada de atención al auto conocimiento y reflexión interior. “(...) l've tried to reach out to people in public space to share more of our ideas to share more of our memories to share more of our anxieties and to share more of our aspirations and all of it is about reimagining the ways that our cities can be built and designed and how our places can better reflect what matters to us as a community and as individuals (...) a lot of my projects are about city communities" (Chang, 2015).

\footnotetext{
1 "La diversidad de las personas que viven en la zona es parte de la esencia de Bankside. Beneficiándose de las olas históricas o del cambio y la migración, la presencia de diferentes colores de piel, lenguas, ocupaciones, estilos de vida y saldos bancarios pueden ser la razón por la que muchos residentes se sienten 'aceptados' aqui' (traducción de la autora).

${ }^{2}$ En el siguiente link se puede visualizar la galería de imágenes de Before I die: http://mergefestival.co.uk/merge2013/2013/9/19/bankside-transformed

3 "Antes de morir quiero..." - en castellano.

4 "Este espacio abandonado se convirtió en uno constructivo (...). Se trata de saber que no estás solo; se trata de entender a nuestros vecinos de maneras nuevas y esclarecedoras; se trata de crear un espacio para la reflexión y la contemplación, y recordar lo que realmente más nos importa a medida que crecemos y cambiamos" (traducción de la autora).

5 “(...) he tratado de llegar a la gente en el espacio público para compartir más de nuestras ideas para compartir más de nuestros recuerdos para compartir más de nuestras ansiedades y compartir más de nuestras aspiraciones y todo esto trata de reimaginar las formas en que nuestras ciudades pueden ser construidas y diseñadas y cómo nuestros lugares pueden reflejar mejor lo que nos importa como comunidad y como individuos (...) muchos de mis proyectos son sobre las comunidades de la ciudad" (traducción de la autora).
} 


\section{HOUSE OF PAIN.}

Los artistas Marcus Lyall y Mark Logue consiguieron convertir para Merge 2013 un edificio abandonado en un espacio en el que las diferentes voces de quienes interactuaban con la instalación dibujaban diversas composiciones de luz. Gracias a las cualidades específicas de cada voz, las luces y las sombras que se podían vislumbrar desde Borough High Street a través de las ventanas del edificio eran únicas e irrepetibles.

Mientras los viandantes que paseaban por la calle se quedaban perplejos al ver esas composiciones lumínicas, los visitantes que se encontraban dentro del edificio escuchaban los gritos que los participantes emitían para dibujar sus propias estructuras creando un ambiente sonoro y lumínico de lo más terrorífico. De esta manera el ciudadano no sólo era un mero espectador de la obra, sino el verdadero creador del espectáculo visual que los viandantes podían disfrutar desde el exterior del edificio ${ }^{6}$.

"Marcus Lyall and Mark Logue of ML Studio had a bright idea and created a place where all your pent-up frustrations can be released with positive repercussions. (...) Marcus and Mark have created an interactive light sculpture in the building where you'll be able to release your stress and the force of your shrieks will generate an instant light display that illuminates the entire building ${ }^{7 \prime \prime}$ (Now. Here. These, 2013: http://now-here-this.timeout.com/2013/09/26/welcome-to-the-house-of-pain/).

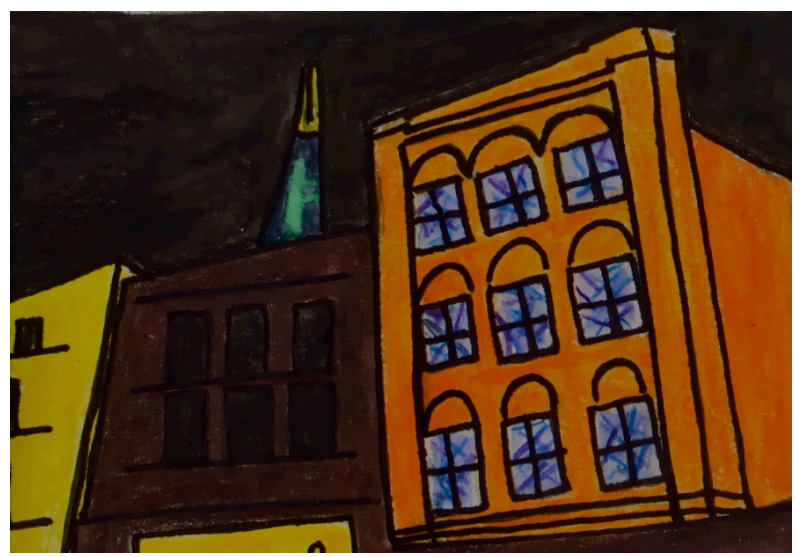

Ilustración 1 - House of Pain, 2016. Boceto realizado a partir de las fotografías de Merge 2013.

Fuente: Estíbaliz Pérez Asperilla.

\section{MINER ON THE MOON Y A POUND OF FLESH FOR 5OP.}

Alex Chinnek convirtió un edificio en desuso y preparado para su cercana demolición en un emplazamiento singular gracias a la creación de una fachada al revés pasando así de ser una construcción olvidada y poco reconocida a una atracción visual que no pasó desapercibida en Blackfriars Road durante el festival. Sin embargo, Alex Chinnek no quiso crear una obra desvinculada de la historia y la esencia del lugar puesto que tuvo en cuenta el pasado del edificio - consistiendo inicialmente en antiguos establos que albergaban caballos y carruajes para su alquiler alrededor del año 1780 y posteriormente sirviendo de almacén para el ganado. De esta forma dio de nuevo vida al inmueble aunque de manera temporal sirviendo incluso de despedida y rememorando su paso por Bankside ${ }^{8}$.

Para el festival del 2014, Chinnek volvió a optar por la fusión entre el pasado y el presente del Distrito a través de la construcción de una casa fabricada con cera rememorando así la antigua fábrica de velas que existía en Bankside dos siglos atrás. A simple vista parecía estar construida a base de ladrillos, pero durante el festival ésta fue deshaciéndose poco a poco como si de una enorme vela se tratara. De esta forma, la obra no era sólo efímera sino que cambiaba a lo largo de los días ofreciendo una evolución constante en la instalación ${ }^{9}$.

\footnotetext{
${ }^{6}$ En el siguiente link se pueden visualizar varias de las interacciones que los usuarios realizaron en the House of Pain: https://www.youtube.com/watch?v=YJzcQEL1reE

7 "Marcus Lyall y Mark Logue de ML Studio tuvieron una idea brillante y crearon un lugar donde todas tus frustraciones contenidas pueden ser liberadas con repercusiones positivas. (...) Marcus y Mark han creado una escultura lumínica interactiva en el edificio donde podrás liberar tu estrés y la fuerza de tus gritos generará una luz instantánea que ilumina todo el edificio" (traducción de la autora).

${ }^{8}$ En el siguiente link se pueden visualizar las fotografías y vídeos de Miner on the Moon: http://mergefestival.co.uk/merge2013/2013/9/19/bankside-transformed

${ }^{9}$ En el siguiente link se puede visualizar la evolución de a Pound of Flesh for 50p: https://www.youtube.com/watch?v=viUYKBBvdBQ
} 
"I think illusions are the distortion of our perception of the physical world that surrounds us, therefore architectural illusions make a lot of sense to me in that respect. Also it's really in tune with the history of art as the re-imagination or the re-presentation of the world that surrounds us (...) ${ }^{10 \prime \prime}$ (Chinnek en Lingham, 2014).

\section{CROSSING STORIES.}

En 2015 Nicolas Henninger dirigió el proyecto denominado como Crossing Stories dentro de una de las acciones de Better Bankside conocida como Bankside Urban Forest consistente en la mejora de la red de calles y espacios públicos en Bankside dando lugar así a su continua regeneración. La comunicación entre el Distrito y su comunidad vuelve a estar presente con las acciones llevadas a cabo dentro del proyecto Crossing stories creando una interacción constante gracias al emplazamiento inesperado de las obras. Bankside se Ilena de textos, preguntas, recuerdos y reflexiones dando la oportunidad de participar de forma activa en el proyecto al poner a disposición un mapa en Menier Gallery para poder recorrer cada una de las localizaciones que formaban parte de esa red de historias y memorias. Tal es el caso de Tony Maas y su River of tomorrow en el túnel victoriano bajo Sourthwark Street donde a través del texto incitaba al lector a reflexionar entre otras cuestiones sobre la inmersión de la sociedad en las nuevas tecnologías creando entre sus líneas un ir y venir entre presente y pasado que provocaba una mayor concienciación de la historia del Distrito ${ }^{11}$.

Otro de los artistas que participó en Crossing stories fue Ruby Wright quien en colaboración con la gente que vivía, trabajaba o visitaba Bankside, llevó a cabo un alfabeto visual. A los/las participantes se les pedía que recordaran un objeto o una persona relacionada con Bankside que tuviera algún valor o significado para ellos - como el Kirkaldy Testing Museum, Crossbones Graveyard o el Borough Market, entre otros - plasmándolo posteriormente en diferentes piezas que ilustraban el suelo y las paredes del Distrito impregnadas no sólo de su historia, sino de los recuerdos de sus colaboradores.
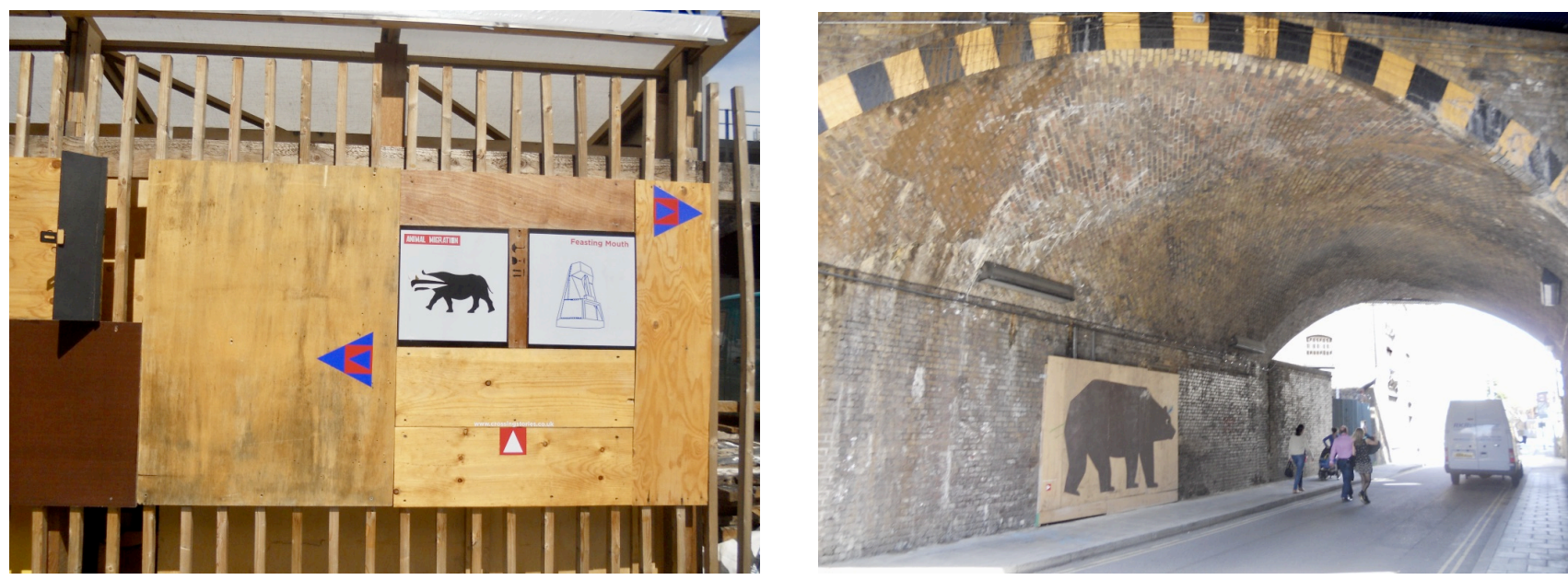

llustraciones 2 y 3 - Algunas de las acciones de Crossing stories que aún se pueden encontrar en las calles de Bankside, 2016. Fuente: Estíbaliz Pérez Asperilla.

\section{CROSSBONES GRAVEYARD.}

Tras realizar una serie de excavaciones arqueológicas en Redcorss Way entre 1991 y 1998 se descubrió la existencia de un antiguo cementerio donde numerosas mujeres que se dedicaron a la prostitución fueron enterradas. Gracias a campañas realizadas por movimientos como Friends of Crossbones Graveyard junto al dramaturgo John Constable se consiguió que se conservara el lugar y se evitara la construcción en dicho emplazamiento consiguiendo posteriormente convertirlo en santuario junto a un jardín conmemorativo.

La continua participación ciudadana ha hecho posible su conservación y desarrollo a través de diferentes vigilias y rituales celebrados mensualmente en su entorno además de las ofrendas que los/las participantes depositan periódicamente en las rejas que dan al exterior. De esta forma, la intervención ciudadana no sólo ha conseguido evitar la destrucción de Crossbones Graveyard, sino la difusión de la historia de todas esas mujeres conocidas como las "Winchester Geese" que tras prohibirles un entierro cristiano debido

\footnotetext{
10 "Creo que las ilusiones son la distorsión de nuestra percepción del mundo físico que nos rodea, por lo tanto las ilusiones arquitectónicas tienen mucho sentido para mí en ese sentido. También está en sintonía con la historia del arte como la re-imaginación o la representación del mundo que nos rodea" (traducción de la autora).

${ }^{11}$ En el siguiente link se puede leer al completo The River of Tomorrow: https://www.crossingstories.co.uk/the-river-of-tomorrow
} 
a su profesión cayeron en el olvido. Incluido incluso en varias guías turísticas, Crossbones Graveyard se ha convertido en una de las visitas indispensables de Londres.
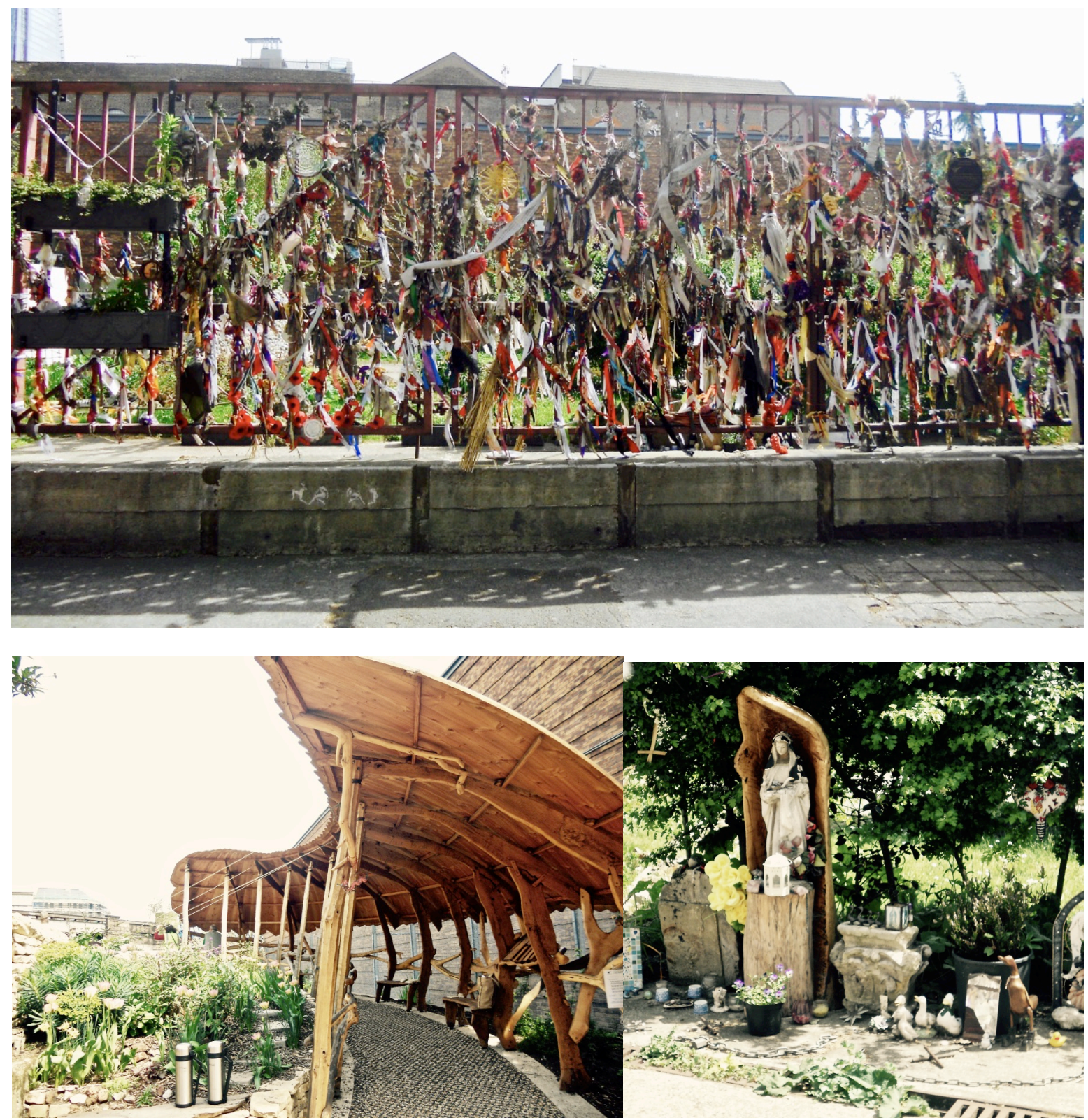

Ilustraciones 4-6 - Crossbones Graveyard (ofrendas, jardín conmemorativo y santuario). Bankside, 2016. Fuente: Estíbaliz Pérez Asperilla.

\section{LIGHT CAPSULES X GHOSTSIGNS.}

Para el London Design Festival 2016 el diseñador Craig Winslow y Sam Roberts llevaron a cabo la instalación Light Capsules $x$ Ghostsigns $^{12}$ donde letreros, anuncios y demás señales que aún se podían vislumbrar vagamente en varios edificios de Bankside aparecieron de nuevo adquiriendo un claro protagonismo frente a los letreros y señales más actuales mediante un excepcional uso de la luz. Tras un estudio exhaustivo de las fotografías de las señales, anuncios y letreros originales, se llevó a cabo una serie de proyecciones sobre su emplazamiento original posibilitando así su idónea visualización.

\footnotetext{
${ }^{12}$ El en siguiente link se pueden visualizar vídeos y fotografías de la instalación Light Capsules $x$ Ghostsigns: http://www.ghostsigns.co.uk/projects/light-capsules
} 
De esta forma, los restos de aquellos letreros, anuncios y señales con cierto carácter fantasmagórico debido a su gran deterioro cobraban vida mediante las nuevas tecnologías dando lugar de nuevo a una fusión entre varios periodos de tiempo que permitía la conservación de parte de la historia y cultura del Distrito. La comunidad local compartía de nuevo experiencias y memorias no sólo gracias a estas instalaciones, sino mediante redes sociales como Twitter, Instagram o Facebook donde subían fotografías y comentarios de las proyecciones dando así no sólo una mayor difusión de la obra, sino permitiendo incluso una gran retroalimentación.

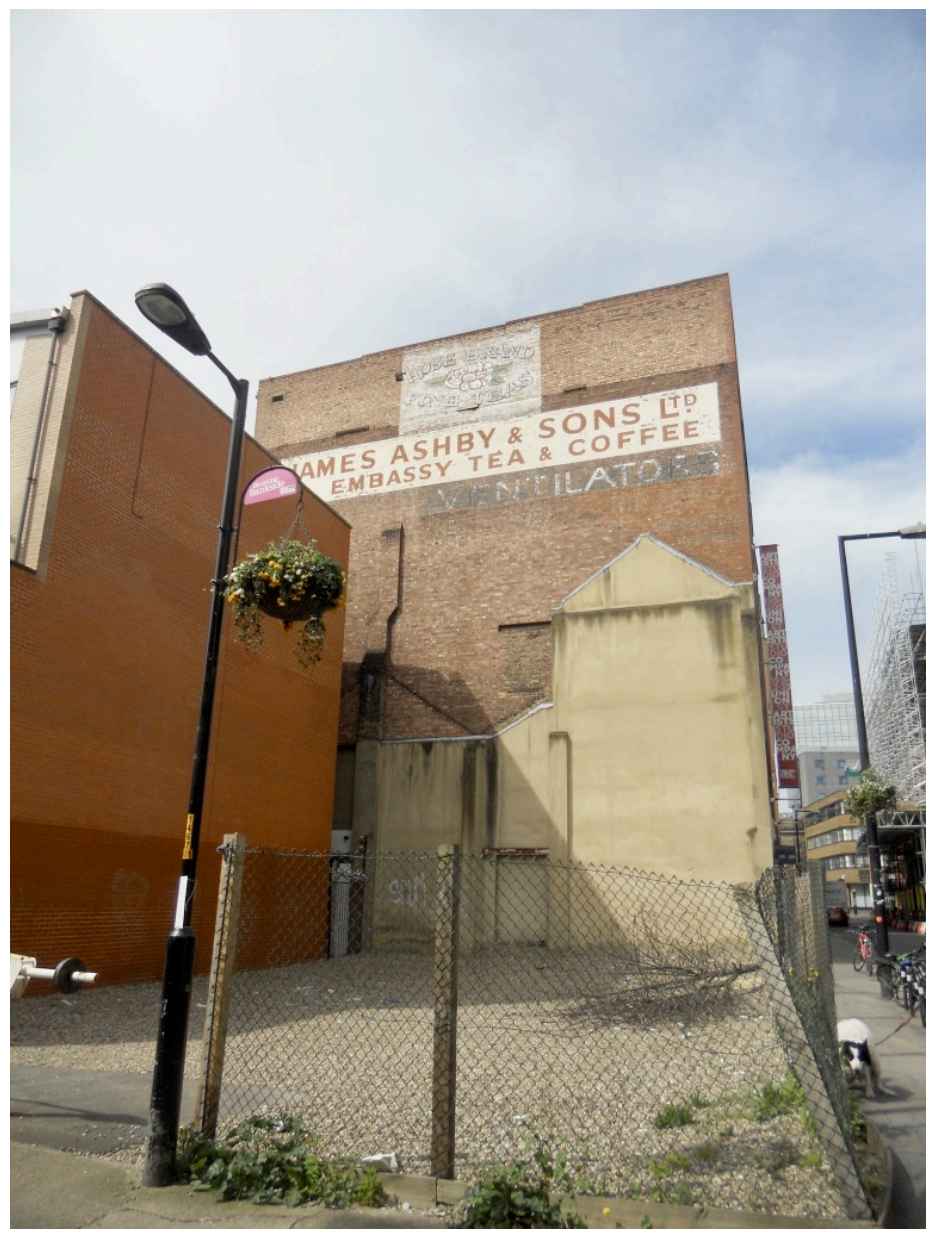

llustración 7 - Ghostsign de Embassy Tea \& Coffee y Rose Brand Fine Teas. Bankside, 2016. Fuente: Estíbaliz Pérez Asperilla.

\section{CONCLUSIONES.}

Las instalaciones y actividades artísticas expuestas en esta comunicación ejemplifican la apuesta por la revitalización de Bankside manteniendo siempre su pasado sin olvidar sus orígenes más oscuros. Aunque muchas zonas y edificios se vayan reconstruyendo y rehabilitando, el pasado y la esencia del Distrito sigue presente creando así un enclave artístico sin igual y con un carácter propio e irrepetible.

La comunidad local se siente parte de Bankside al ser partícipe en todo momento de las acciones culturales y artísticas realizadas compartiendo incluso experiencias y memorias a través de las redes y otras herramientas que los artistas ponen a su disposición. La historia de Bankside y su pasado resurge constantemente en aceras, carreteras, fachadas, túneles y escaparates acompañando a sus habitantes, trabajadores y visitantes en su día a día. Se crea así una comunicación constante entre ciudad e individuo e individuo y ciudad posibilitando a su vez una revitalización de la zona cada vez más enriquecida, puesto que en muchas ocasiones es el público quien conforma el eje principal de las diferentes acciones culturales y artísticas que se llevan a cabo en el Distrito. 
Bankside no se avergüenza de sus orígenes ni de haber sido relacionado con la delincuencia, la prostitución o el pecado en el pasado. La conservación de su historia y el reflejo de ésta en cada una de sus calles, edificios, tabernas y galerías a pesar de haber quedado totalmente desolado tras la fuerte desindustrialización consiguen una revitalización del espacio ejemplar convirtiéndose así en uno de los enclaves artísticos más visitados de Londres.

\section{FUENTES REFERENCIALES.}

Brandon, David y Brooke, Alan: Bankside. London's original district of sin. Amberly, Great Britain, 2011.

Chang, Candy: "Before I die I want too..." En TED Talks, 4, septiembre, 2012 [https://www.youtube.com/watch?v=uebxl|rosiM] [20 de febrero de 2017].

-: "Before I die Wall. Ariba LIVE 2015 Las Vegas". En SAP Ariba, 14, abril, 2015 [https://www.youtube.com/watch?v=_OMQAwLQI3g...] [20 de febrero de 2017].

—-: Página oficial Candy Chang [http://candychang.com] [20 febrero de 2017].

Crossbones: Página oficial de Crossbones [http://crossbones.org.uk] [20 de enero de 2017].

Frearson, Amy: "London building turned upside down by Alex Chinneck". En dezeen, 9, diciembre, 2013. [https://www.dezeen.com/2013/12/06/alex-chinneck-upside-down-building-london/] [15 de febrero de 2017].

Ghostsigns: Light capsules [http://www.ghostsigns.co.uk/projects/light-capsules] [22 de febrero de 2017].

Golden, Grace: Old Bankside. London: Williams \& Norgate LTD, Londres, 1951.

Chinneck, Alex: Página oficial de Alex Chinneck [http://www.alexchinneck.com/] [15 de febrero de 2017].

Henninger, Nicolas: Crossingstories, 2015 [https://www.crossingstories.co.uk] [22 de febrero de 2017].

Jewel, Nichole: "Alex Chinneck's Miner on the Moon Looks Like a Building Flipped on its Head in London". En inhabitat, 12, septiembre, 2013 [http://inhabitat.com/alex-chinnecks-miner-on-the-moon-looks-like-a-building-flipped-on-its-head-in-london/] [15 de febrero de 2017].

Lingham, Daniel: "Size Matters. Interview with Alex Chinnek". En Sculptorvox, Vox 4, 2014 [http://sculptorvox.com/alex-chinneck/] [15 de febrero de 2017].

Merge Festival: Página oficial de Merge Festival [http://mergefestival.co.uk] [22 de febrero de 2017].

Now. Here. These: "Welcome to the House of Pain". En Arts \& Entertainment, Fun London, 26, septiembre, 2013 [http://now-herethis.timeout.com/2013/09/26/welcome-to-the-house-of-pain/] [20 de febrero de 2017].

Pérez Asperilla, Estíbaliz: "Los cinco sentidos en el Distrito Cultural: aplicación del Atlas Multisensorial en Bankside". En Chaves Martín, Miguel Ángel y Lorente, Jesús Pedro (eds.): Barrios Artísticos y Distritos Culturales. Nuevos espacios para la creatividad y la revitalización urbana. Icono 14 Editorial, Madrid, 2016, pp. 229-252. Colabora MINECO Plan Nacional de I+D+i.

Reilly, Leonard y Marshall, Geoff: The story of Bankside. From the River Thames to St. George's Circus. Londres: London Borough of Southwark, Londres, 2001.

Roberts, Howard y Godfrey, Walter H. (1950): Survey of London. Volumen XXII: Bankside (the parishes of St. Saviour and Christchurch Southwark). London County Council, Londres, 1950.

Witherford Watson Mann Architects: Bankside urban forest, s.f. [https://app.box.com/s/3u2q32kta8174qn848yt] [22 de marzo de 2016]. 